\title{
Lesson of the month 1: Septic arthritis with normal acute phase reactants and white cell count in a patient receiving tocilizumab
}

\author{
Authors: Richard Conway, ${ }^{A}$ Carl Orr ${ }^{B}$ and Geraldine M McCarthy ${ }^{C}$
}

\begin{abstract}
Septic arthritis represents $8-27 \%$ of cases of monoarthritis presenting to the emergency department. Tocilizumab is an interleukin- 6 blocking monoclonal antibody with the mechanistic potential to interfere with the body's normal inflammatory response to an infectious insult. We present a case of septic arthritis with a normal white cell count, C-reactive protein, erythrocyte sedimentation rate and fibrinogen in a patient treated with tocilizumab.
\end{abstract}

KEYWORDS: Interleukin-6, septic arthritis, tocilizumab

\section{Case history}

A 70-year-old builder with known rheumatoid arthritis, hypertension, ischaemic heart disease and bilateral total hip replacements presented to the rheumatology clinic with acute monoarthritis of his left knee. Severe pain meant he had great difficulty bearing weight. His rheumatoid arthritis was being treated with methotrexate $20 \mathrm{mg}$ orally and tocilizumab $162 \mathrm{mg}$ subcutaneously weekly and had been in remission at his previous clinic visit. He reported sustaining two superficial grazes on his left knee while kneeling at work on a building site 2 days previously. On examination, there was a large tense left knee effusion (Fig 1). The remainder of the joints were normal and he was apyrexial. White cell count (WCC) was $7.44 \times 10^{9} / \mathrm{L}$, neutrophils $4.77 \times 10^{9} / \mathrm{L}$, erythrocyte sedimentation rate $2 \mathrm{~mm}$ /hour, C-reactive protein $<1 \mathrm{mg} / \mathrm{L}$ and fibrinogen $2.8 \mathrm{~g} / \mathrm{L}$. Aspiration of the left knee yielded $90 \mathrm{cc}$ of group 3 synovial fluid (pyarthrosis). Synovial fluid WCC was $>50,000$, Gram stain was normal and no crystals were identified. The patient was sent to the emergency department with probable septic arthritis but subsequently discharged home with a prescription for an analgesic. This was because the acute phase reactants were normal and it was thus assumed that there was no possibility of infection. After further deterioration,

Authors: ${ }^{A}$ research fellow, Mater Misericordiae University Hospital and CARD Newman Research Fellow, University College Dublin,

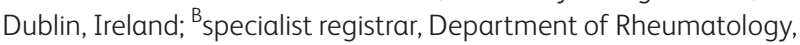
Mater Misericordiae University Hospital, Dublin, Ireland;

${ }^{C}$ consultant rheumatologist, Department of Rheumatology, Mater Misericordiae University Hospital, Dublin, Ireland the patient returned to the rheumatology clinic 2 days later and was admitted directly. Vancomycin and ceftriaxone were commenced for suspected septic arthritis and arthroscopic knee washout performed. Methicillin-sensitive Staphylococcus aureus grew from the synovial fluid culture and antibiotics were rationalised to flucloxacillin. The patient underwent two further knee washouts, in addition to repeated bedside knee aspirations, and was subsequently recommenced on vancomycin because of a lack of clinical improvement. This eventually led to a good treatment response and he was discharged home to complete a 6-week course of intravenous antibiotics.

\section{Discussion}

Septic arthritis is a medical emergency. Delay in treatment results in progressive joint destruction and increased difficulty clearing the infection. Tocilizumab is a humanised monoclonal antibody against the interleukin- 6 receptor. ${ }^{1}$ In common with other biologic agents used in rheumatoid arthritis, tocilizumab is associated with a modest increase in the risk of infection with a relative risk of 1.82 compared with placebo. ${ }^{2}$ Tocilizumab also modulates the systemic response to infection through its inhibitory effect on interleukin- 6 dependent pathways. ${ }^{3}$ Interleukin- 6 is a potent stimulant for the synthesis and release of C-reactive protein and fibrinogen from the liver. Interleukin-6 is also a key participant in both lymphocyte differentiation and neutrophil recruitment. ${ }^{3}$ The inhibition of such a pleiotropic cytokine has the potential to cause significant perturbation in the normal immune response. However, the complete absence of any identifiable systemic immune response - as demonstrated in our case - is distinctly unusual. Despite the lack of elevation in laboratory parameters, our patient mounted a profound local immune response to the infection as shown by the recurrent large volume pyarthrosis. The portal of entry of infection was most likely the grazes overlying the knee joint. Our case highlights the need for sound clinical judgement rather than an overreliance on laboratory tests in assessing for septic arthritis in patients treated with tocilizumab.

\section{Learning points}

$>$ Septic arthritis can occur in the absence of any elevation of WCC, acute phase reactants or fever in tocilizumab-treated patients. 


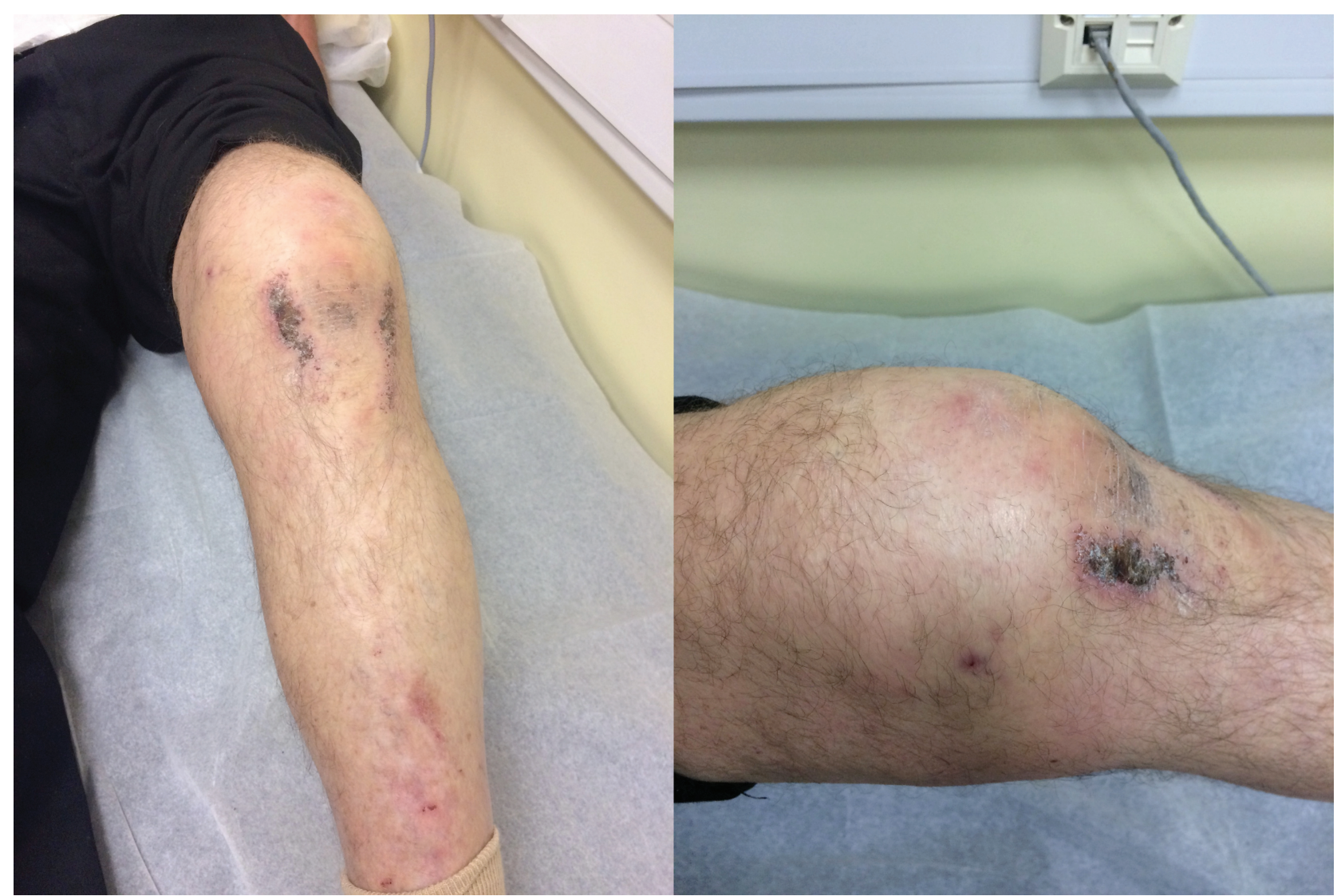

Fig 1. The patient's knee on presentation. A large left knee effusion is visible as are two grazes on the anterior of the knee.

$>$ Clinicians should maintain a high index of suspicion and recognise that lesser degrees of clinical and serological abnormality may herald severe sepsis in tocilizumab-treated patients.

\section{Conflicts of interest}

The authors have no conflicts of interest to declare.

\section{Acknowledgements}

The patient gave permission for publication of the clinical details and images in this article.

\section{References}

1 Woo P, Humphries SE. IL-6 polymorphisms: a useful genetic tool for inflammation research? J Clin Invest 2013;123:1413-4.
2 Strand V, Ahadieh S, French J et al. Systematic review and metaanalysis of serious infections with tofacitinib and biologic diseasemodifying antirheumatic drug treatment in rheumatoid arthritis clinical trials. Arthritis Res Ther 2015;17:362.

3 Rincon M. Interleukin-6: from an inflammatory marker to a target for inflammatory diseases. Trends Immunol 2012;33:571-7.

Address for correspondence: Dr Richard Conway, Department of Rheumatology, Mater Misericordiae University Hospital, Dublin, Ireland.

Email: drrichardconway@gmail.com 